\title{
Parasitaemia and haematological changes in malaria-infected refugees in South Africa
}

\author{
U E Okafor, ${ }^{1}$ BTech, MMedSc; J M Tsoka-Gwegweni, ${ }^{1} \mathrm{MSc}, \mathrm{MPH}, \mathrm{PhD} ; \mathbf{A}$ Bibirigea,${ }^{2} \mathrm{MD} ; \mathrm{A}$ Irimie, ${ }^{2} \mathrm{MD}, \mathrm{PhD} ; \mathbf{C}$ Tomuleasa, ${ }^{2} \mathrm{MD}, \mathrm{PhD}$ \\ ${ }^{1}$ Discipline of Public Health Medicine, School of Nursing and Public Health, College of Health Sciences, Nelson R Mandela School of Medicine, \\ University of KwaZulu-Natal, Durban, South Africa \\ ${ }^{2}$ Department of Haematology, Ion Chiricuta Oncology Institute, Cluj Napoca, Romania, and Iuliu Hatieganu University of Medicine and \\ Pharmacy, Cluj Napoca
}

Correspondingauthor: J M Tsoka-Gwegweni (tsokagwegweni@ukzn.ac.za)

Background. Haematological changes associated with malaria are well recognised, but may vary with level of malaria endemicity and patient background, haemoglobinopathy, nutritional status, demographic factors and malaria immunity. Although malaria in South Africa (SA) has been reduced dramatically in endemic areas, little is known about the haematological changes associated with malaria infection among refugee populations who live in SA cities.

Objective. To describe haematological alterations among malaria-infected refugees living in Durban, SA.

Methods. A cross-sectional study was conducted from September 2012 to July 2013 inclusive at a refugee centre in central Durban. Blood samples from 102 adult black African refugees were examined for infection with malaria parasites, and haematological profiles were compared with standard normal values.

Results. Malaria infection was detected in $16(15.7 \%)$ of the 102 participants. The mean haemoglobin (Hb) value was reduced (mean $9.2 \mathrm{~g} / \mathrm{dL}$ ) in the participants with malaria, who also had an extremely low mean packed cell volume (PCV) of $28.3 \%$. The mean $\mathrm{Hb}$ value in the non-malaria-infected participants was normal $(12.6 \mathrm{~g} / \mathrm{dL})$, and the mean PCV was slightly low $(38.0 \%)$.

Conclusions. Anaemia was more common among participants with malaria infection than among those who were uninfected. Other haematological changes were common in both infected and uninfected participants, suggesting that infections other than malaria, or other underlying factors that cause haematological alterations, may be present. This research needs to be expanded to include a large sample and other areas and infections.

S Afr Med J 2016;106(4):413-416. DOI:10.7196/SAMJ.2016.v106i4.9758

The health burden posed by malaria infection worldwide cannot be overemphasised. ${ }^{[1,2]}$ To date, malaria has represented one of the most severe causes of morbidity among refugee populations worldwide. The United Nations High Commissioner for Refugees reported that this disease affects more than a million cross-border migrants, including women and children. ${ }^{[2]}$ Thirty per cent of the 9.2 million refugees and 25 million displaced persons in the world are in sub-Saharan Africa (SSA). ${ }^{[3]}$ Despite the facts that malaria is a serious health issue for refugees in SSA and that most refugees spend long periods of time in overcrowded conditions, limited research has been conducted to provide accurate data to assist in gaining insight into transmission of the disease, or to curb its spread. ${ }^{[4,5]}$ It has been estimated that approximately $10 \%$ of South Africa (SA)'s population lives in locations considered to be malaria endemic. ${ }^{[6]}$ Approximately $64 \%$ of all cases of malaria reported in SA in 2011 were imported. ${ }^{[6]}$

Malaria is transmitted exclusively through the bite of the anopheles mosquito. Some refugees from non-malaria areas, or areas where the prevalence of malaria is very low, have to travel through malariaendemic areas in order to get to SA. Non-immune refugees are at increased risk and have increased mortality rates, particularly nonimmune children aged under 5 years and non- or semi-immune pregnant women. Refugees who are infected with HIV also fall into the high-risk category. ${ }^{[7]}$ Haematological alterations associated with malaria are well recognised, but specific changes may vary with the level of malaria endemicity and patient background, haemoglobinopathy, nutritional status, demographic factors and malaria immunity. ${ }^{[1]}$
Despite the high number of refugees in SA and the fact that the majority of them come from areas in which malaria is highly endemic, little is known about their health status, particularly with regard to malaria infection and its complications. The objective of this study was to evaluate and determine the frequency of various haematological changes among refugees who were infected with malaria and those who were not infected.

\section{Methods}

The study was an observational, cross-sectional study of refugees living in central Durban, KwaZulu-Natal Province, SA, at a refugee centre run by a church-based non-profit organisation. The study period was from September 2012 to July 2013 inclusive. In total, 102 participants who attended the centre during the data collection period for the study, irrespective of clinical signs or previous tests for malaria, were included. Data on demographic details and previous malaria infection were collected from the participants. Ethical approval was granted by the University of KwaZulu-Natal Biomedical Research Ethics Committee (ref. BE048/14).

Malaria in the participants was confirmed by blood film examination, an SD Bioline malaria rapid diagnostic test (RDT) kit (Standard Diagnostics, Korea) or both. Participants were included in the study if they were were confirmed as positive by the blood film examination or the RDT, whether or not they had clinical symptoms of malaria. Thin and thick blood films were made and stained with Giemsa stain. Malaria parasitaemia was determined by examination under high-power magnification in oil immersion. A manual counter was used to count infected red blood cells in 5 - 10 fields that 
had approximately 1000 red blood cells, all cells that contained parasites in these fields being counted. The RDT used was an antigen-based card test for detecting both Plasmodium falciparum (malaria histidinerich protein II Ag P.f) and other Plasmodium species (Pan, pLDH) (Standard Diagnostics, Korea). The haematological profile of the participants' blood samples was analysed using the Sysmex XE 5000 Automated Haematology Analyser (Sysmex, Canada). The analyser determined the eosinophil count as a percentage of the total differential white blood cell (WBC)count. Eosinophil levels of $1-6 \%$ were defined as within the normal range and levels $>6 \%$ as elevated (eosinophilia).

\section{Results \\ Epidemiological and clinical features}

The study included 102 participants, who were enrolled between September 2012 and July 2013 . The majority (61.8\%) were male, and all originated from African countries known to be malaria endemic; $89.1 \%$ reported to have been infected with the malaria parasite at least once in their countries of origin.

\section{Malaria infection and parasitaemia}

The malaria RDT detected 11 cases among the 102 study participants. Microscopic investigation of thin and thick Giemsastained blood films indicated that five more samples were positive, bringing the total number of positive samples to 16 (overall prevalence of malaria $15.7 \%$ ). Ten test slides indicated infection with $P$. falciparum only, the other six indicating infection with two plasmodium species, $P$. falciparum and $P$. ovale. None of the 16 participants with malaria had classic symptoms such as fever, rigor, chills, sweating or splenomegaly. The remaining participants $(84.3 \%)$ tested negative for malaria infection.

Of the malaria-positive samples, 13 $(81.2 \%)$ were adequate for further parasitaemia analysis. Parasitaemia levels were classified based on the protocol for determining parasitaemia ${ }^{[8]}$ shown in Table 1 . Four participants had a percentage parasitaemia grading level of $0.002 \%$. At this level of grading the average number of parasites was 4075 parasites $/ \mu \mathrm{L}$. The clinical correlations to these values indicate that individuals may be asymptomatic at and below this level. Nine participants had parasitaemia grading levels of $0.2 \%$. Immune individuals are likely to exhibit symptoms above this level. None of the malaria-positive participants had levels of parasitaemia $>2 \%$,

\begin{tabular}{|c|c|c|c|}
\hline $\begin{array}{l}\text { Grading of } \\
\text { parasitaemia, \% }\end{array}$ & $\begin{array}{l}\text { Participants, } n \\
\text { (mean parasites } / \mu \mathrm{L})\end{array}$ & Parasites $/ \mu \mathrm{L}$ & Clinical correlation \\
\hline $0.0001-0.0004$ & - & $5-20$ & - \\
\hline 0.002 & $4\left(\begin{array}{l}4 \\
075\end{array}\right)$ & 100 & $\begin{array}{l}\text { Individuals may be } \\
\text { asymptomatic below this level }\end{array}$ \\
\hline 0.2 & $9(22294)$ & 10000 & $\begin{array}{l}\text { Level above which immune } \\
\text { individuals are likely to } \\
\text { exhibit symptoms }\end{array}$ \\
\hline 2 & - & 100000 & $\begin{array}{l}\text { Maximum parasitaemia for } P \text {. } \\
\text { vivax and } P \text {. ovale }\end{array}$ \\
\hline $2-5$ & - & $100000-250000$ & $\begin{array}{l}\text { Hyperparasitaemia, severe } \\
\text { malaria, increased mortality }\end{array}$ \\
\hline \multirow[t]{2}{*}{10} & - & 500000 & $\begin{array}{l}\text { High mortality, may need } \\
\text { exchange transfusion }\end{array}$ \\
\hline & $\begin{array}{l}\text { Total } N=13 \\
(16342)\end{array}$ & - & - \\
\hline
\end{tabular}

Table 2. Haematological values of the study participants $(N=92)$

\begin{tabular}{llll}
\hline & $\begin{array}{l}\text { Malaria-infected } \\
(\mathbf{N = 6}) \\
\text { mean }(\mathrm{SD})\end{array}$ & $\begin{array}{l}\text { Malaria-negative } \\
(\mathbf{N = 8 6}), \text { mean }(\mathrm{SD})\end{array}$ & $\begin{array}{l}\text { Reference value } \\
(\text { mean }(\mathrm{SD}) \text { or range })\end{array}$ \\
\hline $\mathrm{MAHb}(\mathrm{g} / \mathrm{dL})$ & $9.2(2.05)$ & $12.6(2.3)$ & $13(2)$ \\
Total WBC count $\left(\times 10^{9} / \mathrm{L}\right)$ & $4.1(0.46)$ & $5.6(1.89)$ & $7(3)$ \\
Platelet count $\left(\times 10^{9} / \mathrm{L}\right)$ & $175(140)$ & $227(79)$ & $150-400$ \\
PCV $(\%)$ & $28.3(12.4)$ & $38.0(5.9)$ & $45(5)$ \\
MCV (fl) & $84.3(5.7)$ & $82.0(8.4)$ & $85(9)$ \\
MCH (pg) & $26.1(4.2)$ & $27.0(3.8)$ & $29.5(2.5)$ \\
MCHC (g/dL) & $31.1(5.2)$ & $32.4(1.8)$ & $33(2)$ \\
SD = standard deviation. & & &
\end{tabular}

which is the maximum parasitaemia level for $P$. vivax and $P$. ovale, or hyperparasitaemia levels (Table 1).

\section{Haematological changes}

Ten samples from the 102 participants were not suitable for haematological analysis. Of the remaining 92 samples, 86 were from individuals who tested negative for malaria and only six from individuals who tested positive. Table 2 shows that the mean absolute haemoglobin (MAHb) level was reduced (mean $9.2 \mathrm{~g} / \mathrm{dL}$ ) in the six malaria-infected participants (6/92, $6.5 \%)$, who also had an extremely low mean packed cell volume (PCV) of $28.3 \%$. The MAHb level in the non-malariainfected participants $(86 / 92,93.5 \%)$ was close to normal $(12.6 \mathrm{~g} / \mathrm{dL})$, and the mean PCV was slightly low (38.0\%). The mean total WBC count for the malaria-positive participants was low-normal $(4.1 \times$ $\left.10^{9} / \mathrm{L}\right)$, while the mean for the partici- pants who did not have malaria was normal $\left(5.6 \times 10^{9} / \mathrm{L}\right)$ (Table 2$)$.

The mean corpuscular volume (MCV) was within the normal range in both the malaria-infected (mean $84.3 \mathrm{fl}$ ) and noninfected participants (mean $82.0 \mathrm{fl}$ ), while the mean corpuscular haemoglobin $(\mathrm{MCH})$ was slightly reduced in the malaria-positive participants at an average of $26.1 \mathrm{pg}$, the malaria-negative participants having an average of $27.0 \mathrm{pg}$. The mean corpuscular haemoglobin concentration (MCHC) was slightly reduced in the malaria-infected participants (mean $31.1 \mathrm{~g} / \mathrm{dL}$ ), while the mean for the malaria-negative participants was $32.4 \mathrm{~g} / \mathrm{dL}$ (Table 2).

Of the 92 participants whose $\mathrm{Hb}$ values were evaluated, 35 (38.1\%) were female and 57 (61.9\%) male (Table 3 ). Hb values were graded as normal or indicating moderate or severe anaemia. Overall, the prevalence of anaemia was $63.0 \%(58 / 92)$ and males were the most affected (73.7\%) (Table 3). Twelve 


\begin{tabular}{|c|c|c|c|}
\hline HB levels & $\begin{array}{l}\text { Females } \\
(N=35), n(\%)\end{array}$ & $\begin{array}{l}\text { Males } \\
(N=57), n(\%)\end{array}$ & $\begin{array}{l}\text { Total } \\
(N=92), n(\%)\end{array}$ \\
\hline $\begin{array}{l}\text { Normal } \\
(>12.0 \mathrm{~g} / \mathrm{dL})\end{array}$ & $19(54.3)$ & $15(26.3)$ & $34(37.0)$ \\
\hline $\begin{array}{l}\text { Moderate anaemia } \\
(7.0-11.0 \mathrm{~g} / \mathrm{dL})\end{array}$ & $11(31.4)$ & 37 (64.9) & $48(52.2)$ \\
\hline $\begin{array}{l}\text { Severe anaemia } \\
(<7 \mathrm{~g} / \mathrm{dL})\end{array}$ & $5(14.3)$ & $5(8.8)$ & $10(10.9)$ \\
\hline Total anaemic & $16(45.7)$ & $42(73.7)$ & $58(63.0)$ \\
\hline
\end{tabular}

Table 4. Eosinophil counts of the study participants

\begin{tabular}{lll}
\hline Eosinophil level & $\begin{array}{l}\text { Malaria-infected } \\
(\boldsymbol{N}=\mathbf{6}), \boldsymbol{n}(\%)\end{array}$ & $\begin{array}{l}\text { Malaria-negative } \\
(\boldsymbol{N}=\mathbf{8 6}), \boldsymbol{n}(\%)\end{array}$ \\
\hline High $(>6 \%)$ & $1(16.7)$ & $36(41.9)$ \\
Normal $(1-6 \%)$ & $5(83.3)$ & $50(58.1)$
\end{tabular}

(13.9\%) of the 86 malaria-negative participants had mild to severe thrombocytopenia (platelet count $\left.<150 \times 10^{9} / \mathrm{L}\right)$, while $74(86.0 \%)$ had a normal platelet count (mean $247 \times 10^{9} / \mathrm{L}$ ). Three $(50.0 \%)$ of the malaria-positive participants had moderate thrombocytopenia (20 $100 \times 10^{9} / \mathrm{L}$ ), the remainder having a normal platelet count. Some participants in both the malaria-infected and the malaria-negative groups, particularly the malaria-negative participants, were found to have elevated eosinophil counts ( $16.7 \%$ and $41.9 \%$, respectively). The majority of the participants had normal eosinophil counts (Table 4).

\section{Discussion}

P. falciparum has been and remains the causative agent in $>90 \%$ of malaria cases in SA. ${ }^{[9]} P$. falciparum infection was more common than $P$. ovale infection or mixed infections in our study participants. None of those who were positive for malaria presented with any classic symptoms of malaria disease. Previous studies have linked haematological changes with malaria infection. These changes are often caused by diseases that affect haematopoiesis at various levels, ${ }^{[10]}$ as is the case with malaria.

According to the World Health Organization, anaemia is defined as $\mathrm{Hb}$ values of $<13 \mathrm{~g} / \mathrm{dL}$ in men and $<12 \mathrm{~g} / \mathrm{dL}$ in women. This is based on average $\mathrm{Hb}$ values in healthy individuals. ${ }^{[1]}$ We found that our malaria-infected participants were anaemic, with a low MAHb value of $9.2 \mathrm{~g} / \mathrm{dL}$, which is in agreement with other studies, ${ }^{[1]}$ while the MAHb value for the malaria-negative group was $12.6 \mathrm{~g} / \mathrm{dL}$. However, for some blood tests both malaria-infected and uninfected participants had abnormal results, e.g. low $\mathrm{MCH}$ values in the malaria-positive group and low-normal values in the malarianegative group.

Even in the absence of malaria, nutritional status should be taken into consideration. The morphology of red cells in the presence or absence of malaria can be influenced by nutritional status, e.g. deficiencies in vitamin $B_{12}$, iron or folic acid. ${ }^{[9]}$ In our study, some participants in both the malaria-positive and negative groups had a low PCV. Decreased PCV values can indicate nutritional deficiency. This was also observed by Bhawna et al..$^{[1]}$ in a study investigating haematological changes in malaria.

Co-infection with helminths and malaria can also cause anaemia. Across the African continent, a number of helminth species share their territory with $P$. falciparum, the most common of these being the soil-transmitted helminths Ascaris lumbricoides, Trichuris trichiura and hookworms. ${ }^{[12]}$ Several studies have reported co-infection with P. falciparum and hookworms in a number of populations in Africa.

The effects of infection with one helminth species on the risk of anaemia are well documented, risk being correlated with infection intensity. Hookworm causes iron deficiency anaemia through the process of intestinal blood loss. Schistosomes also cause blood loss, as their eggs penetrate the intestinal wall. Like malaria, schistosomiasis can also cause anaemia as a result of destruction of red blood cells and dyserythropoiesis. ${ }^{[1]}$ On the basis of the specific mechanisms by which malaria, hookworms and schistosomes reduce haemoglobin levels, it can be speculated that interaction due to their combined presence could increase the risk of anaemia. ${ }^{[2]}$ Future studies on co-infections with malaria and helminths and nutritional status in our study setting could explain the high level of anaemia found in this population.

Leucocytosis and leucopenia have been reported in patients with malaria, ${ }^{[1,10]}$ although controversy in this regard seems to exist. ${ }^{[1]}$ In the present study, mean WBC counts in the malaria-positive and negative groups were near-normal and normal, respectively. This is in line with other studies. ${ }^{[1]}$ However, normal WBC counts are not enough to clearly indicate freedom from underlying disease. A differential analysis of the individual WBC counts would have given a more precise blood picture. The elevated eosinophil counts in both groups may have indicated intestinal parasite infestation. Few studies have investigated parasitic infestation among refugees in settings like ours.

None of the malaria-positive participants presented with any common malaria symptoms, i.e. they had asymptomatic malaria. The clinical correlations with malaria parasitaemia grading values indicate that at the low levels observed patients may be asymptomatic, and this may explain why our participants had no clinical signs of malaria.

We also found that our malaria-positive participants had thrombocytopenia, which is in line with several other studies from other African settings endemic for malaria. ${ }^{[6]}$

\section{Study limitations}

The laboratory methods used in our study to test for malaria are known to be less sensitive for detecting asymptomatic than symptomatic infections, so the prevalence of malaria in our subjects may have been underestimated. The polymerase chain reaction would have provided a better estimate of malaria infection than RDT and microscopy. ${ }^{[13]}$ The study provided a description of parasitaemia and haematological parameters in a small sample of refugee population. Research using an analytical design and a powered sample size would provide a better understanding of the relationship between malaria and the related haematological parameters.

\section{Conclusion}

All our malaria-positive participants were asymptomatic carriers, as all had low-grade parasitaemia and none presented with any obvious malaria symptoms. The malaria-positive participants also had severe anaemia, and thrombocytopenia. The low PCVs could indicate nutritional deficiency. Similarly, the low $\mathrm{MCH}$ values confirm anaemia, and could also indicate haemoglobinopathy or nutritional deficiency. The elevated eosinophil counts observed in $>40 \%$ of the participants could have resulted from infection with malaria, intestinal parasite infestation, or other undiagnosed health burdens. As is done in high-income countries, we recommend regular health screening and 
adequate treatment of refugee populations to reduce disease burden and transmission in KwaZulu-Natal and SA as a whole.

Authorship. JMT-G was the principal investigator and takes primary responsibility for the paper. UEO performed the laboratory work, the research nurse and UEO were responsible for recruiting participants into the study, data analysis was performed by UEO and JMT-G, and the article was written by UEO, JMT-G, AB, AI and CT.

\section{References}

1. Bhawna S, Bharthi A, Yogesh K, Reena A. Parasitemia and hematological alterations in malaria: study from the highly affected zones. Iran J Path 2013;8(1):1-8.

2. United Nations High Commissioner for Refugees. UNHCR: Country operations profile. South Africa: 2012. http://www.unhcr.org/egi-bin/texts/vtx/page (accessed 12 July 2012).

3. Seicean A. Refugee health making a difference in Sub-Saharan Africa: A literature review. 2008. www://cwru.edu/med/epidbio/mphp439/refugee_health.pdf (accessed 7 March 2012).

4. Bayoh N, Akhwale W, Ombok M, et al. Malaria in Kaukuma refugee camp, Turkana, Kenya: Facilitation of Anopheles arabiensis vector population by installed water distribution and catchment systems. Malar J 2011:10(149):1475-2875. DOI:10.1186/1475-2875-10-149
5. Montavlo G, Renal-Querron M. Fighting against malaria: Prevent wars while waiting for the malaria vaccine. 2007. http://www.econ.upf.edu/ (accessed 5 June 2012)

6. Maharaj J, Morrison N, Seocharan I, et al. The feasibility of malaria elimination in South Africa. Malar J 2012;11:423-428. DOI:10.1186/1475-2875-11-423

7. World Health Organization. WHO areas. http://www.who.int/media centre (accessed 23 November 2013).

8. Garcia LS. Diagnostic Medical Parasitology. 4th ed. Washington, DC: ASM Press, 2001.

9. Maharaj R, Raman J, Moonsar D, et al. Epidemiology of malaria in South Africa: From control to elimination. S Afr Med J 2013;103(10):779-788. DOI:10.7196/SAMJ.7441

10. Maina R, Walsh D, Gaddy C, et al. The impact of Plasmodium falciparum infection on haematological parameters in children living in Western Kenya. Malar J 2010;9(Suppl 3):S4. DOI:10.1186/1475-28759-S3-S4

1. Aina B, Suman V, Fairbanks V, et al. Incidence of anaemia in older people: An epidemiological study in a well defined population. J Am Geriatr Soc 1997;45 (7):825-831.

12. Brookers S, Willis A, Pullan R, et al. Epidemiology of plasmodium helminth co-infection in Africa: Populations at risk, potential impact on anaemia and prospects for combining a control. Am J Trop Med Hyg 2007;77(66 Suppl):88-98.

13. Ndao M, Bandyayera E, Kokosin E, et al. Comparison of blood smear, antigen detection, and nested-PCR methods for screening from regions where malaria is endemic after a and nested-PCR methods for screening from regions where malaria is endemic after a
malaria outbreak in Quebec, Canada. J Clin Microbiol 2004;42(6):2694-2700. DOI:10.1128/ JCM.42.6.2694-2700.2004

Accepted 22 October 2015 